\title{
Efficient Learning in Sparsely Connected Boltzmann Machines
}

\author{
Marcel J. Nijman and Hilbert J. Kappen \\ RWCP$^{\star}$ Novel Functions, SNN ${ }^{\star \star}$ Laboratory \\ Dept. of Medical Physics and Biophysics, University of Nijmegen \\ Geert Grooteplein 21, NL 6525 EZ Nijmegen, The Netherlands
}

\begin{abstract}
We present a heuristical procedure for efficient estimation of the partition function in the Boltzmann distribution. The resulting speed-up is of immediate relevance for the speed-up of Boltzmann Machine learning rules, especially for networks with a sparse connectivity.
\end{abstract}

\section{Introduction}

Boltzmann Machines (BMs) [1] form an attractive group of Neural Networks for several reasons. The local learning rule, for instance, offers the possibility of parallel implementation. Their main disadvantage, however, is that computing the correlations $\left\langle S_{i} S_{j}\right\rangle$ exactly can only be done in a reasonable time for small networks. Although the correlations can be approximated with simulated annealing, this is very slow. Some good results have been reported about mean field learning, which speeds up the computation by approximating $\left\langle S_{i} S_{j}\right\rangle$ by $\left\langle S_{i}\right\rangle\left\langle S_{j}\right\rangle$. However, in most situations mean field learning leads to large errors and cannot be applied successfully. An approach which leads to tractable Boltzmann Machines is to restrict the state space by arranging the hidden units into layers that perform a winner-take-all competition [4] or many-take-all [9].

For some simple structures efficient learning rules exist. In [8] a decimation method is presented which leads to linear time learning rules for Boltzmann Trees. In Section 3 we explain the principle of decimation. Since tree-structured networks are of limited use, we would like to extend this method to apply to networks with a general architecture. This can be done as follows. For any network structure we can clamp some nodes, such that the remaining structure is a tree, which can be decimated. From this we can compute the partition function, as is shown in Section 4. The challenge is to identify a small set of nodes which to clamp. This problem is addressed in Section 5. The learning rules which result from this are very efficient when applied to networks with a sparse connectivity, as is shown in Section 6. Some extensions are discussed in Section 7. We finish with the conclusions in Section 8.

We are interested in Boltzmann Machines with sparse connectivity for several reasons. The use of sparse connectivity for Bayesian networks is well appreciated [7], and is argued to play an important role in probabilistic reasoning,

\footnotetext{
* Real World Computing Partnership

$\star \star$ Dutch Foundation for Neural Networks
} 
as it makes many conditional independencies explicit, and facilitates interpretation, inference, and estimation and storage of the parameters. Also, prior knowledge concerning conditional independencies can be incorporated into the structure of the network. We believe that most of the motivations for using sparse connectivity in Bayesian networks also applies to Boltzmann Machines.

\section{Boltzmann Machine Learning}

Consider a Boltzmann Machine with binary units $S_{i}$. The probability to observe a state $S=\left\{S_{i}\right\}$ is

$$
p(S)=\frac{1}{Z} \exp (-H(S))
$$

with

$$
H(S)=-\sum_{(i j)} w_{i j} S_{i} S_{j}
$$

and

$$
Z=\sum_{S} \exp (-H(S))
$$

Learning is defined as gradient descent on the Kullback-Leibler divergence [6] and leads to the following learning rule [1]

$$
\Delta w_{i j} \propto{\overline{\left\langle S_{i} S_{j}\right\rangle_{\text {clamped }}}}\left\langle S_{i} S_{j}\right\rangle_{\text {free }} .
$$

Since the two-point correlations are related to the partition function by $\left\langle S_{i} S_{j}\right\rangle=$ $\frac{\partial}{\partial w_{i j}} \ln Z$, efficient computation of $Z$ leads to efficient learning rules. Therefore, we will only concentrate on computing the partition function $Z$. Computing this partition functions by explicit summing over all the states of $S$ involves an exponential number of terms, and is thus intractable in general. However, for some architectures more efficient learning rules exits, as we shall see.

\section{Decimation}

Recently an algorithm has been described [8] which computes the partition function of a BM with a tree-structure in linear time (for the free and the clamped phase). The algorithm is based on decimation [3], and can be described as follows.

Consider the two network fragments in Figure 1. In the left fragment $S_{2}$ and $S_{3}$ have an arbitrary number of connections with the rest of the network. $S_{1}$, however, is only connected to $S_{2}$ and $S_{3}$. By integrating out $S_{1}$ we obtain the network on the right. This introduces an additional weight $w_{23}$ into the network. In order for the two networks to have the same probability distribution on the remaining units the following equation must hold:

$$
\sum_{S_{1}} \exp \left(w_{12} S_{1} S_{2}+w_{13} S_{1} S_{3}\right)=\sqrt{C} \cdot \exp \left(w_{23} S_{2} S_{3}\right)
$$



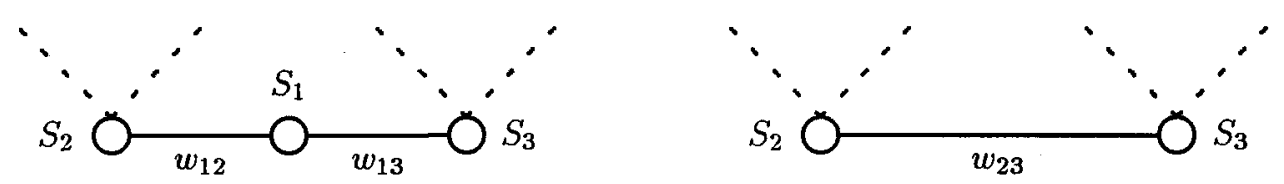

Fig. 1. Two network fragments. The right fragment is obtained from the left fragment by integrating the probability distribution over $S_{1}$.

for $S_{2}= \pm 1$ and $S_{3}= \pm 1$. The solution to this is [8]

$$
\begin{aligned}
\tanh w_{23} & =\tanh w_{12} \tanh w_{13} \\
C & =2 \cosh \left(w_{12}+w_{13}\right) \cdot 2 \cosh \left(w_{12}-w_{13}\right) .
\end{aligned}
$$

For a BM with a tree-structure all units can be decimated out. This can be shown as follows. Introduce a bias unit $S_{0}$ permanently fixed to 1 . Let $w_{i 0}$ represent the bias of $S_{i}$. Handle units which should be clamped by leaving them unclamped, but setting the bias $w_{i 0}$ to $\pm \infty$. The resulting structure will not be a tree anymore, because all units are connected to the same bias unit. However, a leaf in the original tree now has degree 2 (it is connected to its parent, and to the bias unit) and thus can be decimated out. This can be done iteratively until all the units have been decimated out. Thus, for a BM with a tree-structure the partition function (and thus the correlations $\left\langle S_{i} S_{j}\right\rangle$ ) can be computed exactly in linear time.

\section{Divide and Conquer}

The idea of decimation can be applied to a general BM in the following way. Partition the units $S$ in two sets $X$ and $Y$ such that $G_{Y}$ (the sub-graph defined on $Y$ ) contains no cycles. A trivial choice is $Y=\emptyset$ and $X=S$, but in general more interesting partitionings exist. If the units in $X$ are clamped we can decimate the units in $Y$, since $G_{Y}$ is a forest. We can rewrite (1) as follows

$$
p(X, Y)=\frac{1}{Z} \exp (-H(X)-H(Y ; X))
$$

with

$$
H(X)=-\sum_{(i j)} w_{i j} X_{i} X_{j}
$$

and

$$
H(Y ; X)=-\sum_{(i j)} w_{i j} Y_{i} Y_{j}-\sum_{(i j)} w_{i j} Y_{i} X_{j},
$$

where the partition function is

$$
\begin{aligned}
Z & =\sum_{X} \exp (-H(X)) \sum_{Y} \exp (-H(Y ; X)) \\
& =\sum_{X} Z_{Y ; X} .
\end{aligned}
$$



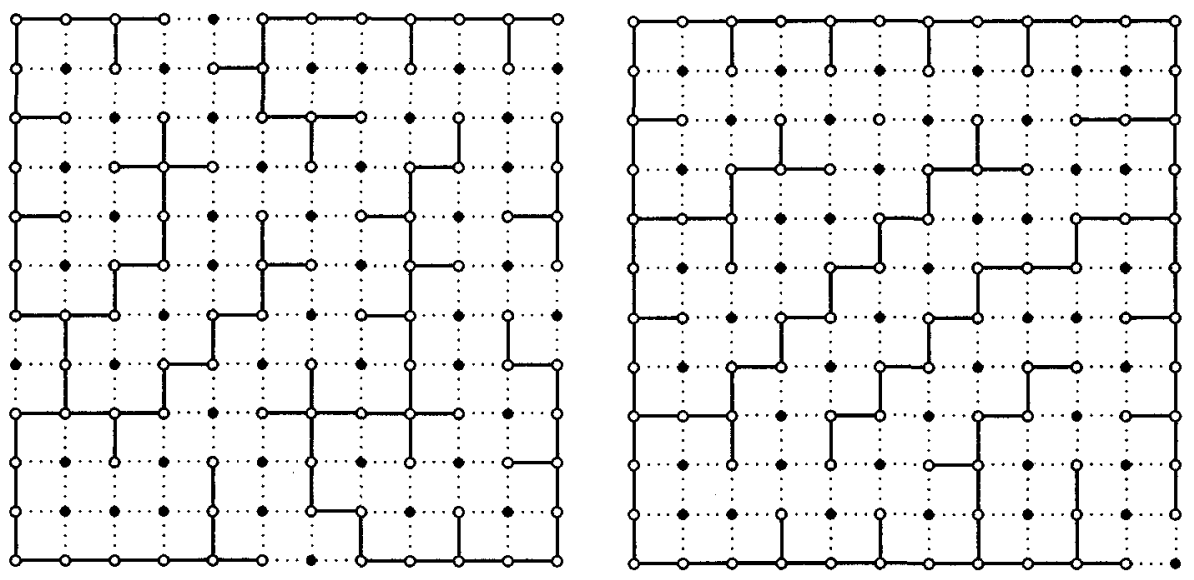

Fig. 2. Two partitionings of a 12 by 12 grid. The black units cut every cycle of the grid. The partitioning on the left was obtained with our heuristic. The partitioning on the right is optimal (to our knowledge).

Note that Eq. 11 holds independent of the partitioning (even if $G_{Y}$ contains cycles), but that only something has been gained if $Z_{Y ; X}$ can be computed efficiently.

So, the original partition function $Z$ can be computed by summing over the states of the units in $X$, and computing $Z_{Y ; X}$ (in linear time) for each state of $X$. If $X$ has a limited size, this sum is tractable, and the partition function can be computed exactly. If $X$ is a large set, we can estimate $Z$ with Gibbs sampling by using

$$
Z=2^{|X|}\left\langle Z_{Y ; X}^{-1}\right\rangle_{X}^{-1}
$$

\section{Feedback Vertex Set}

The efficiency of Eq. 11 or Eq. 12 depends on the size of $X$; the smaller the better. Therefore, we need an algorithm to identify a small set $X$. Such a set which cuts every cycle in a network is known as a feedback vertex set [2]. So, our optimization problem becomes a search for the minimal feedback vertex set, which, unfortunately, is NP-complete [5]. Therefore, we use the following heuristical algorithm. Start with an empty set $X$ and with a graph representing the network structure. Prune the graph by iteratively deleting all nodes with degree less than 2 (leaves and unconnected roots). Then, identify the node with the highest degree, delete it from the graph, and insert it in the set $X$. Repeat this procedure until the graph contains no more nodes. This heuristic is fast (linear in the number of nodes), and generally gives a small set $X$. As an example, consider the two networks in Figure 2. The network on the left shows a typical partitioning obtained with our algorithm on a 12 by 12 grid. The set $X$ consists of the black units, and the dotted lines symbolize the biases 


\begin{tabular}{||c|c|c|c||}
\hline edges & Equation 13 & Equation 12 & deleted nodes \\
\hline 100 & $218.7 \pm 7.2$ & $19.11 \pm 0.51$ & $11.678 \pm 0.033$ \\
200 & $222.6 \pm 7.9$ & $90.9 \pm 2.9$ & $22.036 \pm 0.034$ \\
400 & $232.8 \pm 7.7$ & $227.9 \pm 6.5$ & $31.287 \pm 0.033$ \\
800 & $227.3 \pm 6.7$ & $231.1 \pm 7.3$ & $38.159 \pm 0.025$ \\
\hline
\end{tabular}

Table 1. Average number of iteration needed for convergence for two methods of computing the partition function for a Boltzmann machine with 50 nodes, and the average number of nodes deleted, plus error bounds on the estimates.

which result from clamping the black units. This partitioning (with $|X|=46$ ) is quite good, since we believe the optimum (shown on the right in Figure 2) to be 45 . Typically, our heuristic finds solutions with 46 to 48 black units. Therefore, although finding the optimal set is NP-hard we can still find a good approximation with this heuristic.

\section{Numerical Results}

It is to be expected that Gibbs sampling with Eq. 12 converges faster than Gibbs sampling on the total space $S$ using Eq. 13.

$$
Z=2^{|S|}\langle\exp (H(S))\rangle_{S}^{-1} .
$$

In order to show the significance of this gain we have generated random BMs with 50 nodes by randomly inserting a number of edges $(100,200,400$, and $800)$. All edges were given a weight uniformly drawn from $[-1,1]$. Then, we computed a feedback vertex set $X$ and estimated the partition function with both methods. We stopped when the standard deviation on the estimate was less than $1 \%$ of $Z$. Table 1 shows for both algorithms and for a varying number of edges the number of iterations needed until convergence. The results are averaged over 1000 networks. We conclude that the results of standard Gibbs sampling are hardly effected by the complexity of the network, whereas the new method leads to much faster convergence for sparse networks.

\section{Discussion}

In this paper we have derive learning rules for sparsely connected networks. We have not addressed the problem of identifying the structure of a sparce network which fits a given data set well.

Secondly, we note that $G_{Y}$ doesn't have to be a forest. Decimation cannot only be used on trees, but also on a bit more complex structures like rings and ladders. This suggests a heuristical algorithm to determine $X$ by decimating the original network as far as possible, deleting the node with the highest degree, and so on. 
Another observation is that the efficiency of the resulting algorithm is not only determined by the number of units in $X$, but also by the number of connections in $G_{X}$. Less connections means that Gibbs sampling will sample the state space of $X$ more evenly. How this can be incorporated in an algorithm to compute a set $X$ is still an open question.

\section{Conclusions}

We have shown how the partition function of a Boltzmann Machine with a sparsely connected structure can be computed efficiently, which leads to efficient learning rules. This allows for a trade-off between the complexity of a network (and, consequently, the complexity of the problems that can be solved) and the computation time that one is willing to spend on training the network.

\section{References}

1. D.H. Ackley, G.E. Hinton, and T.J. Sejnowski. A learning algorithm for Boltzmann Machines. Cognitive Science, 9:147-169, 1985.

2. M.R. Garey and D.S. Johnson. Computers and Intractability: A Guide to the Theory of NP-Completeness. Freeman, San Francisco, 1979.

3. C. Itzykson and J. Drouffe. Statistical Field Theory. Cambrigde University Press, Cambridge, 1991.

4. B. Kappen and M.J. Nijman. Radial basis Boltzmann machines and learning with missing values. In Proceedings of the World Congress on Neural Networks, volume I, pages 72-75. Lawrence Erlbaum Associates, Mahwah, NJ, 1995.

5. R.M. Karp. Reducibility among combinatorial problems. In R.E. Miller and J.W. Thatcher, editors, Complexity of Computer Computations, pages 85-103. Plenum Press, 1972.

6. S. Kullback. Information theory and statistics. Wiley, N.Y., 1959.

7. J. Pearl. Probabilistic reasoning using graphs. In B. Bouchon and R.R. Yager, editors, Uncertainty in Knowledge-Based Systems, pages 200-202. Springer-Verlag, 1987.

8. L. Saul and M. Jordan. Learning in Boltzmann trees. Neural Computation, 6(6):1174-1184, 1994.

9. D.M.J. Tax and H.J. Kappen. Learning structure with many-take-all networks. In proceedings of ICANN, 1996. 